\title{
Is It Cost Effective to Obtain Fungal and Acid-Fast Bacillus Cultures during Spine Debridement?
}

\author{
Mark J. Lambrechts ${ }^{1}$, Devin D. St. Clair ${ }^{1}$, Jinpu Li ${ }^{1}$, James L. Cook ${ }^{1,2}$, Bradley S. Spence ${ }^{1}$, \\ Emily V. Leary ${ }^{1}$, Theodore J. Choma ${ }^{1}$, Donald K. Moore ${ }^{1}$, Christina L. Goldstein ${ }^{3}$ \\ ${ }^{I}$ Department of Orthopaedic Surgery, University of Missouri, Columbia, MO, USA \\ ${ }^{2}$ Thompson Laboratory for Regenerative Orthopaedics, University of Missouri, Columbia, MO, USA \\ ${ }^{3}$ Department of Orthopaedic Spine Surgery, University of Colorado at Colorado Springs, Colorado Springs, CO, USA
}

\begin{abstract}
Study Design: Retrospective study.
Purpose: To identify the rate of positive acid-fast bacillus (AFB) and fungal cultures during spine debridement, determine whether these infections are more common in certain spine segments, identify comorbidities associated with these infections, and determine whether the universal performance of fungal and AFB cultures during spine debridement is cost effective.

Overview of Literature: Spine infections are associated with significant morbidity and costs. Spine fungal and AFB infections are rare, but their incidence has not been well documented. As such, guidance regarding sample procurement for AFB and fungal cultures is lacking.

Methods: A retrospective review of medical record data from patients undergoing spine irrigation and debridement (I\&D) at the University of Missouri over a 10-year period was performed.

Results: For patients undergoing spine I\&D, there was a $4 \%$ incidence of fungal infection and $0.49 \%$ rate of AFB infection. Steroid use was associated with a higher likelihood (odds ratio, 5.62; 95\% confidence interval, 1.33-23.75) of positive fungal or AFB cultures. Although not significant, patients undergoing multiple I\&D procedures had higher rates of positive fungal cultures during each subsequent I\&D. Over a 10-year period, if fungal cultures are obtained for each patient, it would cost our healthcare system $\$ 12,151.58$. This is compared to an average cost of $\$ 177,297.64$ per missed fungal infection requiring subsequent treatment.

Conclusions: Spine fungal infections occur infrequently at a rate of $4 \%$. Physicians should strongly consider obtaining samples for fungal cultures in patients undergoing spine I\&D, especially those using steroids and those undergoing multiple I\&Ds. Our AFB culture rates mirror the false positive rates seen in previous orthopedic literature. It is unlikely to be cost effective to send for AFB cultures in areas with low endemic rates of AFB.
\end{abstract}

Keywords: Antifungal agents; Bacillus; Cost-benefit analysis; Cost-savings; Fungi; Lumbar spine; Surgical wound infection

\section{Introduction}

Surgical site infections (SSIs) are relatively uncommon complications after spinal surgery, with a reported incidence of $2.1 \%$ based on a database study of 108,419 patients [1]. However, spine SSIs are associated with

Received Apr 29, 2021; Revised May 31, 2021; Accepted Jul 11, 2021

Corresponding author: Mark J. Lambrechts

Missouri Orthopaedic Institute, University of Missouri, 1100 Virginia Ave., Columbia, MO 65212, USA

Tel: +1-573-882-5731, Fax: +1-573-882-8200, E-mail: lambrechtsm@health.missouri.edu 
significant morbidity and can result in catastrophic outcomes for patients. Risk factors for SSI have been well documented and include modifiable patient-related risk factors (i.e., body mass index [BMI], diabetes, malnutrition, and tobacco use) and non-modifiable risk factors (i.e., rheumatoid arthritis, revision surgery, and age) [25]. Identifying and mitigating these risk factors are crucial for providers, as spine SSIs cost twice as many healthcare dollars as uninfected cases $(\$ 37,009$ versus $\$ 16,227)$ with cost per quality-adjusted life year exceeding three times that of uninfected patients $(\$ 185,045$ versus $\$ 55,955)$ [6]. As such, SSI prevention has a tremendous benefit to patients and the healthcare system. Bacterial infections in the spine have been well characterized such that preventative strategies have been developed and implemented. However, fungal SSIs have not been well delineated with respect to their incidence and risk factors. Clinical characterization of fungal SSIs is critical for developing more effective preventative and management strategies.

When performing spine irrigation and debridement (I\&D), the typical clinical mantra is to obtain samples for microbial cultures, fungal cultures, and acid-fast bacillus (AFB) cultures. However, this approach is not based on robust data to support its clinical utility or costeffectiveness. Therefore, the present study was initiated to determine the relative incidence and cost-effectiveness of performing anaerobic, fungal, and AFB cultures for each patient undergoing spine I\&D at the University of Missouri. The study was designed to test the hypothesis that the universal performance of fungal and AFB cultures would be cost effective based on the culture costs relative to the costs of missing these infections. The experimental design allowed for (1) identifying the incidence of fungal and AFB infections in this patient cohort, (2) comparing the incidence of fungal and AFB infections in cervical, thoracic, and lumbar spine segments, (3) characterizing relevant risk factors for fungal and AFB SSIs, and (4) determining the cost of requesting cultures for all patients who undergo spinal I\&D procedures.

\section{Materials and Methods}

\section{Patient population}

The study was approved by by the Institutional Review Board of the University of Missouri (IRB review no., 2014081) and our project was given active-exempt status under exempt category 45 CFR 46.104d(4). Due to our exempt status and retrospective study design, informed consent was not obtained for individual patients. Inclusion criteria included all patients who underwent spine I\&D by orthopedic spine surgeons at the University of Missouri between January 2008 and December 2018. These patients were identified based on the following current procedural terminology (CPT) codes: 110140, 110180, 11042, 11043, 11044,22010 , and 22015. The CPT codes corresponded to hematoma/seroma drainage, I\&D of complex wound, debridement of subcutaneous tissue, muscle, fascia, or bone, and I\&D of deep abscess of the cervical, thoracic, and lumbar spine. Owing to government funding, our spine surgeons frequently manage referred, medically complex, and uninsured patients, potentially yielding a higher rate of AFB- or fungus-positive cases.

Patients were excluded from the study if no samples associated with the spine I\&D procedure were submitted for microbial, fungal, or AFB culture. For patients meeting the inclusion criteria, the medical comorbidities, number of spine I\&Ds, and antifungal use were extracted from the electronic medical record for statistical analyses.

\section{Financial costs}

All hospital-related costs associated with microbial cultures, I\&D procedures, physician fees, and facility costs were determined using administrative data during the years 2014 to 2019. These costs were further divided into physician fees (spine surgeon, anesthesiologist, medical co-management physician, and infectious disease specialist) and facility costs (standard spine X-rays and advanced imaging, laboratory costs for metabolic and inflammatory panels, glucose tests, operating room time and personnel, physical therapy, and nursing fees). The cost of a missed fungal infection was calculated as the total cost (physician fees and hospital costs) of the actual course of treatment of the fungal infection. The cost of each culture was determined and assigned based on the current cost of a culture, as well as the Medicare reimbursement rate for culture.

\section{Analysis}

Rates of medical comorbidities (categorical) were analyzed using either the chi-square test or Fisher's exact test to determine associations with positive fungal cultures. A two-sample $t$-test was used to determine the significance 
of differences between the mean age for those with and without positive fungal cultures. Chi-square tests were conducted to determine whether the index or subsequent surgeries had increased rates of AFB or fungal infections and to determine whether there was a greater rate of fungal or AFB infection in the cervical, thoracic, thoracolumbar, or lumbosacral region of the spine. The cost of a fungal/AFB culture at our institution in 2020 was $\$ 69.20$, with Medicare reimbursement equal to $\$ 9.34$. Cost savings to the healthcare system were determined by subtracting the cost of testing all patients who receive spine I\&D, less the conservative Medicare reimbursement rate, from the combined physician fees and facility costs associated with a patient with a fungal infection.

\section{Results}

A total of 254 patients were identified, of whom 52 were excluded. All of these 52 patients were excluded because no culture samples were sent to the lab due to the assumption of a lack of infection. The cohort used for all analyses was therefore composed of 202 patients undergoing 271 spine I\&Ds (Table 1). The average age was $53.1 \pm 15.9$ years, with $51.5 \%$ identifying as female. For the majority

Table 1. Demographics of patients with positive fungal culture compared to negative fungal cultures

\begin{tabular}{lll} 
& \multicolumn{2}{c}{ Fungal culture } \\
\cline { 2 - 3 } Characteristic & $\begin{array}{c}\text { Negative } \\
(\mathrm{n}=193)\end{array}$ & $\begin{array}{c}\text { Positive } \\
(\mathrm{n}=8)\end{array}$ \\
\hline Age $(\mathrm{yr})$ & $53.1 \pm 15.8$ & $52.6 \pm 15.9$ \\
\hline Female (\%) & 51.8 & 50 \\
\hline I\&D location & & \\
\hline Cervical & $26(17.6)$ & $2(33.3)$ \\
\hline Thoracic & $4(2.7)$ & 0 \\
\hline Thoracolumbar & $11(7.4)$ & $1(16.7)$ \\
\hline \multicolumn{1}{c}{ Lumbosacral } & $107(72.3)$ & $3(50.0)$ \\
\hline I\&D is an index procedure. & $45(23.6)$ & $2(25.0)$ \\
\hline I\&D is a subsequent procedure prior to spine surgery & & \\
\hline Anterior spine fusion & $24(16.2)$ & $2(33.3)$ \\
\hline Posterior spine fusion & $70(47.3)$ & $3(50.0)$ \\
\hline Anteroposterior spine fusion & $1(0.7)$ & 0 \\
\hline Spine decompression & $53(35.8)$ & $1(16.7)$ \\
\hline
\end{tabular}

Values are presented as mean \pm standard deviation or number $(\%)$, unless otherwise stated.

I\&D, irrigation and debridement. of patients (77.4\%), the I\&D was performed at the site of a previous spine surgery, whereas the I\&D was the first spine surgery for 46 (22.6\%) of the 202 patients. Overall, 28 patients had two spine I\&Ds, 10 had three I\&Ds, and seven patients had four I\&Ds.

Eight patients had spine fungal infections with a total of 10 positive cultures due to one patient having three consecutive positive fungal cultures. One patient had a concomitant AFB infection. The fungal SSI rate was $4 \%$ and the AFB SSI rate was $0.49 \%$ for this patient population. Interestingly, only six of these patients were treated with antifungals. Patients were determined to have cleared the infection once serial inflammatory markers showed a declining trend and there was no further purulence or other signs of infection during the spine debridement. This happened after the inclusion of the antifungal regimen in all six of the patients who were treated with antifungals. The two patients not treated with antifungals had concomitant bacterial SSIs and were treated with appropriate antibiotics. Of the remaining six patients treated with antifungals, one had concomitant multimicrobial bacterial SSI and AFB SSI, one had a concomitant methicillin-sensitive Staphylococcus aureus infection, and the remaining four had isolated fungal SSIs. None of the patients with positive fungal cultures were readmitted to the hospital for continued spine infection after treatment and subsequent hospital discharge. Patient follow-up after hospital discharge was recorded if they saw either the patient's primary physician or a spine attending and averaged 21 months (range, 8-45 months). One patient died after 8 months due to medical factors unrelated to the spine infection. There were no significant differences with respect to age $(p=0.94)$ or sex $(p=1.00)$ between patients with and without fungal SSIs.

With respect to risk factors associated with spine fungal SSIs, use of oral steroids was strongly associated with obtaining a positive fungal culture (odds ratio, 5.62; 95\% confidence interval, 1.33-23.75). No other assessed comorbidities were significantly associated with a fungal SSI (Table 2); however, the association of chronic obstructive pulmonary disease (COPD) was almost statistically significant $(p=0.05)$. The rate of obtaining a positive fungal culture increased with each subsequent I\&D; however, this association was also not statistically significant $(p=0.07)$ (Table 3). Further, there were no statistically significant associations with positive fungal cultures and I\&D location (i.e., in the cervical, thoracic, thoracolumbar, or lumbar/ 
Table 2. Relationship of comorbidities to positive fungal infections

\begin{tabular}{|c|c|c|c|c|}
\hline \multirow{2}{*}{ Independent variable } & \multirow{2}{*}{ Levels } & \multicolumn{2}{|c|}{ Fungus or AFB positive } & \multirow{2}{*}{$p$-value } \\
\hline & & No & Yes & \\
\hline \multirow[t]{2}{*}{ Sex } & Male & 94 (95.92) & $4(4.08)$ & 1.0000 \\
\hline & Female & $100(96.15)$ & $4(3.85)$ & \\
\hline \multirow[t]{2}{*}{ Obesity } & No & $103(94.50)$ & $6(5.50)$ & 0.2935 \\
\hline & Yes & 90 (97.83) & $2(2.17)$ & \\
\hline \multirow[t]{2}{*}{ Hypertension } & No & $104(97.20)$ & $3(2.80)$ & 0.4774 \\
\hline & Yes & 89 (94.68) & $5(5.32)$ & \\
\hline \multirow[t]{2}{*}{ COPD } & No & $172(97.18)$ & $5(2.82)$ & 0.0509 \\
\hline & Yes & 20 (86.96) & $3(13.04)$ & \\
\hline \multirow[t]{2}{*}{ Asthma } & No & $179(96.76)$ & $6(3.24)$ & 0.1256 \\
\hline & Yes & $14(87.50)$ & $2(12.50)$ & \\
\hline \multirow[t]{2}{*}{ Diabetes } & No & 140 (95.89) & $6(4.11)$ & 1.0000 \\
\hline & Yes & 53 (96.36) & $2(3.64)$ & \\
\hline \multirow[t]{2}{*}{ Depression } & No & $122(95.31)$ & $6(4.69)$ & 0.7132 \\
\hline & Yes & $71(97.26)$ & $2(2.74)$ & \\
\hline \multirow[t]{2}{*}{ Thyroid disease } & No & 162 (95.86) & $7(4.14)$ & 1.0000 \\
\hline & Yes & 31 (96.88) & $1(3.12)$ & \\
\hline \multirow[t]{2}{*}{ Congestive heart failure } & No & $186(95.88)$ & $8(4.12)$ & 1.0000 \\
\hline & Yes & $7(100.00)$ & 0 & \\
\hline \multirow[t]{2}{*}{$\begin{array}{l}\text { Peripheral vascular } \\
\text { disease }\end{array}$} & No & $180(95.74)$ & $8(4.26)$ & 1.0000 \\
\hline & Yes & $13(100.00)$ & 0 & \\
\hline \multirow[t]{2}{*}{ Autoimmune disease } & No & $179(96.24)$ & $7(3.76)$ & 0.4685 \\
\hline & Yes & 14 (93.33) & $1(6.67)$ & \\
\hline \multirow[t]{2}{*}{ Taking oral steroids } & No & $163(97.60)$ & $4(2.40)$ & $0.0268^{\mathrm{al}}$ \\
\hline & Yes & $29(87.88)$ & $4(12.12)$ & \\
\hline \multirow[t]{2}{*}{ Chronic kidney disease } & No & $184(95.83)$ & $8(4.17)$ & 1.0000 \\
\hline & Yes & $8(100.00)$ & 0 & \\
\hline \multirow[t]{2}{*}{ Liver disease } & No & $174(95.60)$ & $8(4.40)$ & 1.0000 \\
\hline & Yes & $19(100.00)$ & 0 & \\
\hline \multirow[t]{2}{*}{ History of DVT } & No & $183(95.81)$ & $8(4.19)$ & 1.0000 \\
\hline & Yes & $10(100.00)$ & 0 & \\
\hline \multirow[t]{2}{*}{ Smoking status } & No & $154(95.65)$ & $7(4.35)$ & 1.0000 \\
\hline & Yes & $39(97.50)$ & $1(2.50)$ & \\
\hline \multirow[t]{2}{*}{ Active cancer } & No & $180(96.26)$ & $7(3.74)$ & 0.4447 \\
\hline & Yes & 13 (92.86) & $1(7.14)$ & \\
\hline
\end{tabular}

Values are presented as number (\%), unless otherwise stated.

AFB, acid-fast bacteria; COPD, chronic obstructive pulmonary disease; DVT, deep vein thrombosis

al 0 dds ratio $=5.6207$
Table 3. Rate of infections based on number of spine I\&D procedures

\begin{tabular}{lccccc} 
Infection & I\&D \#1 & I\&D \#2 & I\&D \#3 & I\&D \#4 & $p$-value \\
\hline Fungal infection & & & & & 0.0673 \\
\hline Not infected & 196 & 42 & 14 & 5 & \\
\hline Infected & 5 & 2 & 2 & 1 & \\
$\quad$ Infection rate (\%) & 2.49 & 4.40 & 12.50 & 16.67 & \\
\hline AFB infection & & & & & 0.9556 \\
\hline Not infected & 201 & 44 & 16 & 5 & \\
\hline Infected & 1 & 0 & 0 & 0 & \\
\hline Infection rate (\%) & 0.50 & 0 & 0 & 0 & \\
\hline Fungal or AFB infection & & & & & 0.1133 \\
\hline Not infected & 196 & 42 & 14 & 5 & \\
\hline Infected & 6 & 2 & 2 & 1 & \\
\hline Infection rate (\%) & 2.97 & 4.50 & 12.50 & 16.67 & \\
\hline
\end{tabular}

I\&D, irrigation and debridement; AFB, acid-fast bacteria.

lumbosacral region of the spine) (Table 4).

The average physician fees alone, without accounting for facility fees during hospital admission, for spine I\&D with culture were $\$ 19,409$, with total average physician fees for inpatient care at $\$ 20,257$ and outpatient care at $\$ 2,440.62$. Both physician fees and facility costs were then separately tabulated for the eight patients with fungal infection. The average cost for each patient with a fungal infection was $\$ 161,875.67$ in facility costs and $\$ 27,977.87$ in physician fees (Table 5).

Additionally, we considered a scenario in which no infections were missed, all patients had cultures, and patient treatment was guided by culture results (our current practice). Conversely, we also considered a scenario in which no cultures were taken and all patients had a missed fungal infection requiring subsequent readmission to the hospital. The difference in the associated costs, minus the reimbursed Medicare cost, was used as the calculated cost savings for our healthcare system. This was calculated to be $\$ 1,406,229.52$.

\section{Discussion}

The results of the present review of medical record data from patients undergoing spine I\&D at our orthopedic hospital over a 10 -year period revealed a $4 \%$ incidence of fungal SSIs and a $0.49 \%$ incidence of AFB SSIs. Oral steroid use was associated with a five times higher likelihood of positive fungal and AFB cultures. Furthermore, there were no significant differences in the likelihood of 
Table 4. Rate of fungal infection based on location of spine I\&D

\begin{tabular}{|c|c|c|c|c|c|}
\hline Infection & Cervical & Thoracic & Lumbar/sacral & Thoracolumbar & $p$-value \\
\hline Fungal infection & & & & & 0.5760 \\
\hline Not infected & 26 & 4 & 107 & 11 & \\
\hline Infected & 2 & 0 & 3 & 1 & \\
\hline Infection rate (\%) & 7.14 & 0 & 2.73 & 8.33 & \\
\hline AFB infection & & & & & - \\
\hline Not infected & 28 & 4 & 111 & 12 & \\
\hline Infected & 0 & 0 & 1 & 0 & \\
\hline Infection rate (\%) & 0 & 0 & 0.71 & 0 & \\
\hline Fungal or AFB infection & & & & & 0.5691 \\
\hline Not infected & 26 & 4 & 108 & 11 & \\
\hline Infected & 2 & 0 & 3 & 1 & \\
\hline Infection rate (\%) & 7.14 & 0 & 2.70 & 8.33 & \\
\hline
\end{tabular}

I\&D, irrigation and debridement; AFB, acid-fast bacteria.

Table 5. Relative costs of universal performance of fungal testing versus total costs (physician fees and facility costs) for missed fungal infections

\begin{tabular}{llrr} 
Scenario & \multicolumn{1}{c}{ Type } & Average cost (\$) & Total cost (\$) \\
\hline Fungal infections missed requiring subsequent treatment & Facility cost & $152,246.75$ & $1,217,974.03$ \\
& Physician fees & $25,050.88$ & $200,407.07$ \\
\hline Testing all patients & Cost to system (overall average) & $177,297.64$ & $1,418,381.10$ \\
\hline & Testing costs & 69.20 & $14,047.60$ \\
\hline & Reimbursed (if all Medicare) & 9.34 & $1,896.02$ \\
\hline
\end{tabular}

obtaining positive fungal cultures based on the anatomical location of the I\&D within the spine. We were able to determine that the financial burden to our healthcare system is substantially smaller if fungal cultures are obtained for all patients at every I\&D over a 10 -year period $(\$ 12,151.58)$ than if no fungal cultures are obtained leading to missed infections requiring treatment (cumulative cost for all patients of $\$ 1,418,381.10$ ). This indicates that fungal cultures can be considered cost effective and may decrease morbidity and the fiscal burden of not identifying fungal infections.

Spine infections are associated with significant morbidity and costs. Spine bacterial SSIs have been studied extensively; however, there has been minimal research guiding best practice management of when to determine if a patient should have a fungal culture sent to microbiology. The most relevant data available relate to fungal and AFB cultures from aseptic total joint revisions, which were associated with reported "false positive" rates of $1.6 \%$ for fungus and $0.5 \%$ for AFB [7]. The $4 \%$ incidence of positive fungal cultures for spine SSIs in the present study is much higher than the rate of false positives reported by Tokarski et al. [7]. However, it is possible that at least two of our positive fungal cultures were false positives based on the apparent resolution of the SSI without antifungal treatment or further procedures. The $0.49 \%$ incidence of positive AFB cultures for spine SSIs in the present study matches the AFB false positive rate reported by Tokarski et al. [7] for aseptic total joint revisions. Although the patient was treated with long-term antibiotics for a presumed atypical mycobacterial infection, the patient also had three consecutive positive fungal cultures and consecutive positive polymicrobial bacterial cultures. As such, it seems plausible that the single AFB culture was a false positive.

In general, the common practice for spine surgeons when performing spine I\&D is to obtain samples for microbial cultures and "send for everything." Spine SSIs are 
associated with tremendous costs [6], such that identification of risk factors, prevention, and mitigation of costs for these infections are critically important to patients and the healthcare system. Risk factors for bacterial SSIs have been well documented and include modifiable patientrelated risk factors (i.e., BMI, diabetes, malnutrition, and tobacco use) and non-modifiable risk factors (i.e., rheumatoid arthritis, revision surgery, and age) [2-5]. While oral steroid use was the only risk factor identified as having a significant association with the presence of fungal spine infection in this study, the risk factors associated with bacterial SSI should still be considered until more definitive data are available.

Our finding of an increased risk for fungal infections with steroid use aligns with previous literature identifying steroid use as a risk factor for such infections [8]. However, owing to the substantial patient morbidity and the tremendous financial burden associated with missing an infection, we recommend obtaining fungal cultures in all patients undergoing spine I\&D regardless of their risk factors. At institutions with very low levels of AFB infection, the rate of false positive AFB infections may be greater than the rate of true AFB infections. In these instances, it may not be cost effective to send for AFB culture for patients with a suspected spine infection.

There are limitations that should be considered when interpreting and applying the results of the present study. First, we excluded 52 patients, representing $20.5 \%$ of our study population, due to no cultures being sent to microbiology. Since these patients did not undergo additional surgeries, it can likely be safely assumed that these were uninfected cases. This does falsely elevate the proposed effectiveness of sending fungal cultures for each I\&D. However, owing to the low overall costs of obtaining fungal cultures, this would not change the outcome on whether it is cost effective to obtain fungal cultures. In fact, this would strengthen our argument that AFB cultures are unlikely to be cost effective in areas with low endemic rates of AFB. In addition, our methods for estimating the cost of a missed fungal infection requiring subsequent treatment are likely at the upper limit of what a missed infection would cost. However, the total cost to our healthcare system of obtaining fungal cultures over a 10 -year period $(\$ 12,151.58)$ is well below the average combined physician's fee $(\$ 19,409)$ for a single patient requiring spine debridement. Further, costs across the period of study were not adjusted for inflation and only current costs for cultures were considered. Finally, the numbers of spine fungal and AFB infections identified at our Midwest university hospital were low and hospitals with larger numbers of fungal or AFB infections may identify additional risk factors for identifying spine fungal and AFB infections. However, even with these limitations, our data appear to definitively support obtaining fungal cultures in all patients undergoing spine I\&D regardless of their risk factors. The utility of obtaining routine AFB cultures is dependent on the endemic rates of AFB.

\section{Conclusions}

Our retrospective medical record-based review demonstrated that it is cost effective to send for fungal cultures during each spine I\&D. Over a 10 -year period at our institution, the costs of sending for fungal cultures would amount to a hospital cost of $\$ 12,151.58$ compared with $\$ 1,418,381.10$ if all fungal infections were missed and required subsequent treatment. The use of steroids was a risk factor for obtaining positive fungal cultures, while COPD ( $p=0.05)$ and each subsequent I\&D $(p=0.07)$ trended toward significance for identifying a fungal infection. The data regarding AFB cultures are less clear. The rate of previously reported false positive AFB cultures in aseptic total joint revisions mirrors the rate of AFB-positive cultures found in our cohort of patients undergoing spine debridement. Therefore, sending for AFB cultures is unlikely to be cost effective in patients requiring spine I\&D at institutions with low endemic rates of AFB.

\section{Conflict of Interest}

No potential conflict of interest relevant to this article was reported.

\section{Acknowledgments}

We would like to thank Kylee Rucinski, BS, MHA and Shannon Kiehl, BS, MS, for gathering all financial data related to facility costs and physician fees included in this manuscript.

\section{Author Contributions}

All authors have read and approved the final submitted manuscript. The following is the author contribution: 
MJL, DDSC, JL, JLC, BSS, EVL, TJC, DKM, CLG: substantial contributions to research design, acquisition, analysis and interpretation of data; MJL, DDSC, JL, JLC, BSS, EVL, TJC, DKM, CLG: drafting the paper and revising it critically; and MJL, DDSC, JL, JLC, BSS, EVL, TJC, DKM, CLG: approval of the submitted and final versions.

\section{References}

1. Smith JS, Shaffrey CI, Sansur CA, et al. Rates of infection after spine surgery based on 108,419 procedures: a report from the Scoliosis Research Society Morbidity and Mortality Committee. Spine (Phila Pa 1976) 2011;36:556-63.

2. Olsen MA, Nepple JJ, Riew KD, et al. Risk factors for surgical site infection following orthopaedic spinal operations. J Bone Joint Surg Am 2008;90:62-9.

3. Meng F, Cao J, Meng X. Risk factors for surgical site infections following spinal surgery. J Clin Neurosci 2015;22:1862-6.
4. Yao R, Zhou H, Choma TJ, Kwon BK, Street J. Surgical site infection in spine surgery: who is at risk? Global Spine J 2018;8(4 Suppl):5S-30S.

5. Janssen DM, van Kuijk SM, d'Aumerie B, Willems P. A prediction model of surgical site infection after instrumented thoracolumbar spine surgery in adults. Eur Spine J 2019;28:775-82.

6. Pennington Z, Sundar SJ, Lubelski D, Alvin MD, Benzel EC, Mroz TE. Cost and quality of life outcome analysis of postoperative infections after posterior lumbar decompression and fusion. J Clin Neurosci 2019;68:105-10.

7. Tokarski AT, O’Neil J, Deirmengian CA, Ferguson J, Deirmengian GK. The routine use of atypical cultures in presumed aseptic revisions is unnecessary. Clin Orthop Relat Res 2013;471:3171-7.

8. Wu J, Keeley A, Mallen C, Morgan AW, PujadesRodriguez M. Incidence of infections associated with oral glucocorticoid dose in people diagnosed with polymyalgia rheumatica or giant cell arteritis: a cohort study in England. CMAJ 2019;191:E680-8. 